\title{
Prevalência de diabetes melito e tolerância à glicose diminuída nos indígenas da Aldeia Jaguapiru, Brasil
}

\author{
Geraldo Ferreira de Oliveira, ${ }^{1}$ Teresinha Regina Ribeiro de Oliveira, ${ }^{1}$ \\ Fernanda França Rodrigues, ${ }^{1}$ Lincoln Ferreira Corrêa, ${ }^{1}$ \\ Adauto Tsutomu Ikejiri ${ }^{1}$ e Luiz Augusto Casulari ${ }^{2}$
}

Como citar Oliveira GF, Oliveira TRR, Rodrigues FF, Corrêa LF, Ikejiri AT, Casulari LA. Prevalência de diabetes melito e tolerância à glicose diminuída nos indígenas da Aldeia Jaguapiru, Brasil. Rev Panam Salud Publica. 2011:29(5):315-21.

RESUMO Objetivo. Avaliar a prevalência de diabetes melito (DM) e de tolerância à glicose diminuída em indígenas da Aldeia Jaguapiru, em Dourados, Estado de Mato Grosso do Sul.

Métodos. Foram avaliados indígenas de 18 a 69 anos de idade entre agosto de 2007 e julho de 2008. A amostra aleatória simples foi obtida pelo sorteio de 349 de 1255 casas da Aldeia. Excluiram-se as mulheres grávidas, os indivíduos não indígenas e seus descendentes moradores na Aldeia, além dos usuários de glicocorticoide. A amostra incluiu 606 pessoas, 268 homens e 338 mulheres. Realizaram-se dosagens da glicemia capilar com glicosimetro e teste de tolerância oral à glicose, quando necessário.

Resultados. A prevalência de DM foi de 4,5\%, e a de tolerância diminuída à glicose, de 2,2\%, com maior frequência entre as mulheres. Dos diabéticos, 44,4\% não tinham diagnóstico. A obesidade esteve presente em 14,2\% dos homens e em 30,8\% das mulheres. A prevalência de hipertensão arterial foi de 29,7\% entre todos os sujeitos participantes e de $67,5 \%$ entre os diabéticos e os indivíduos com tolerância à glicose diminuída. Não foi encontrada associação estatística entre fumar e presença de DM ou de tolerância à glicose diminuída.

Conclusões. As prevalências de DM e de tolerância à glicose diminuída foram inferiores nesta amostra em relação à população brasileira; entretanto, a prevalência de obesidade foi maior e a de hipertensão arterial foi semelhante. São recomendáveis orientações nutricionais e incentivo à prática de atividades físicas entre os indígenas da Aldeia Jaguapiru como forma de prevenção do DM.

Palavras-chave

Diabetes melito; população indígena; hipertensão; obesidade; índios sul-americanos; Brasil.

O diabetes melito (DM) compreende um grupo heterogêneo de distúrbios metabólicos de etiologias variadas, resultantes da secreção deficiente de insulina

\footnotetext{
1 Universidade Federal da Grande Dourados, Faculdade de Ciências da Saúde, Curso de Medicina, Dourados (MS), Brasil. Correspondência: Geraldo Ferreira de Oliveira, gfor@brturbo.com.br

2 Universitário de Brasília, Serviço de Endocrinologia, Brasília (DF), Brasil.
}

pelas células beta-pancreáticas, da resistência periférica à insulina ou de ambas. É marcado pela presença de hiperglicemia crônica e por alterações no metabolismo das proteínas e das gorduras. Um estado pré-diabético, ou tolerância à glicose diminuída, é atribuído aos indivíduos com glicemia de 140 a 199 $\mathrm{mg} / \mathrm{dL}$ após 2 horas no teste oral de tolerância à glicose (TOTG) (1).
Globalmente, o DM alcança proporções epidêmicas. Pelos custos elevados, que envolvem o controle e o tratamento de suas complicações, tornou-se um crescente problema para a saúde pública (2). Atualmente, existem mais de 285 milhões de diabéticos nos cinco continentes; aproximadamente $90 \%$ são do tipo 2, que envolve resistência ou produção diminuída de insulina, e 10\% são 
do tipo 1, no qual a insulina não é produzida (3). Cerca de 197 milhões de indivíduos em todo o mundo têm tolerância à glicose diminuída. Estima-se que, em 2025, esse número chegará a 420 milhões de pessoas (4). Um estudo multicêntrico realizado em nove capitais brasileiras entre os anos de 1986 e 1988 relatou uma prevalência de $7,6 \%$ de diabetes e de $7,8 \%$ de tolerância à glicose diminuída em pessoas de 30 a 69 anos de idade (5).

Até o início do século XX, o DM era uma doença rara na população nativa das Américas. A partir de 1940, apareceram relatos de DM na comunidade indígena norte-americana, na qual, atualmente, a prevalência de DM supera aquela reportada para a população geral $(6,7)$. Contudo, as taxas de prevalência do DM nas populações indígenas das Américas têm variação ampla, de 4,4\% na população adulta dos índios otomi (8) do México até mais de $50 \%$ na população acima de 35 anos entre os índios pima nos Estados Unidos (7). Entre aborígenes australianos, a prevalência de DM é maior do que na população não indígena. Além disso, tanto a doença como as suas complicações macro e microvasculares têm início mais precoce nessa população (9).

A primeira referência a DM em grupos indígenas no Brasil é dos anos 1970, entre os índios caripunas e palikures, no Amapá. Esse estudo realizou 192 glicemias de jejum em participantes acima de 12 anos; dois indivíduos apresentaram nível acima de $200 \mathrm{mg} / \mathrm{dL}$ e sintomatologia clássica de DM (10). Um estudo realizado na comunidade ianomâmi com 72 indígenas com idade acima de 18 anos encontrou apenas uma glicemia capilar pós-prandial maior do que $200 \mathrm{mg} / \mathrm{dL}$ (11). Alguns estudos relataram o aumento da prevalência de DM tipo 2, obesidade e doença cardiovascular em vários grupos indígenas (12-14).

A transição epidemiológica e a emergência de morbimortalidade relacionada às doenças crônicas não transmissíveis entre os indígenas são atribuídas às alterações no estilo de vida desses povos e têm exigido atenção, treinamento e recursos especiais para ações de prevenção, diagnóstico e tratamento (15). Essas alterações são marcantes na Reserva Indígena de Dourados, localizada a 5 quilômetros da zona urbana do município com o mesmo nome. Abriga as etnias guarani, kaiowá e terena e sofre com o grande adensamento populacio- nal, com a deterioração de sua organização socioeconômica, com as mudanças de hábitos alimentares e com a degradação do ecossistema, que se manifesta no total desaparecimento das matas nativas e dos animais silvestres e no consequente comprometimento das atividades de subsistência desses indígenas. A assimilação de hábitos e costumes da população do entorno não implicou aumento da miscigenação, pois é raro o casamento entre índios e não índios dentro das duas aldeias da Reserva de Dourados (16).

O presente estudo teve por objetivo detectar a prevalência de DM e de tolerância à glicose diminuída e sua relação com fatores de risco cardiovasculares nos indivíduos de 18 a 69 anos de idade na Aldeia Jaguapiru, localizada na Reserva de Dourados.

\section{MATERIAIS E MÉTODOS}

O presente estudo transversal investigou a prevalência do DM e da tolerância à glicose diminuída na Aldeia Jaguapiru, Estado de Mato Grosso do Sul. A coleta de dados foi realizada no período de agosto de 2007 a julho de 2008. Residiam na Aldeia, na época do estudo, 5727 pessoas, sendo 2578 adultos com idade entre 18 e 69 anos (1 286 mulheres e 1292 homens) (16).

A amostra foi calculada considerandose uma prevalência de diabetes de $2,2 \%$ na Aldeia Buriti (17), localizada no mesmo Estado, e que apresenta características semelhantes às da Aldeia Jaguapiru. O nível de confiança estabelecido foi de 5 desvios padrão, e o erro máximo permitido foi de 3 . Com base nesses parâmetros, verificamos que o número mínimo da amostra seria de 485 indivíduos entre 18 e 69 anos.

Existem 1255 casas na Aldeia, ou seja, uma média de 2,05 adultos por residência. Como a intenção era avaliar em torno de $25 \%$ da população elegível para a pesquisa, sorteamos inicialmente 350 moradias.

A amostra aleatória simples foi obtida por meio do seguinte processo: 1) anotação dos números oficiais de todas as 1255 casas da Aldeia em pedaços de papel que foram dobrados e colocados em uma urna; 2) retirada da urna de 350 papéis contendo os números das residências que seriam as escolhidas para fazer parte da pesquisa. Todas as vezes que, nas residências selecionadas, não houvesse indivíduos cujas características preenchessem os critérios de inclusão na pesquisa, o procedimento foi repetido tal como descrito acima, até que se completasse a amostra. Os fatores de exclusão foram uso atual de glicocorticoide, gravidez em qualquer faixa etária e não ser indígena. Todos os indígenas com idade de 18 a 69 anos residentes nas 350 casas sorteadas que não preenchiam esses critérios de exclusão foram convidados a participar do estudo. Como uma das casas sorteadas foi demolida, foram coletados dados em 349 casas.

$\mathrm{Na}$ primeira visita ao domicílio dos participantes, foram colhidas, por meio de um formulário, informações sobre condições socioeconômicas, hábitos alimentares, atividade física, consumo de bebida alcoólica e cigarro, idade, sexo, ocupação, nível educacional e histórico pessoal e familiar de DM e hipertensão. A idade considerada para cada indivíduo foi aquela registrada na identificação da Fundação Nacional do Índio (FUNAI).

Na segunda visita ao domicílio, após jejum de 12 horas, dosou-se a glicemia capilar com um glicosímetro, com tira reagente de leitura rápida (glicoseoxidase). As glicemias de 70 a $99 \mathrm{mg} / \mathrm{dL}$ foram consideradas normais; as de 100 a $125 \mathrm{mg} / \mathrm{dL}$ foram consideradas como glicemia de jejum alterada. Os sujeitos com glicemia capilar de jejum de 126 $\mathrm{mg} / \mathrm{dL}$ a $199 \mathrm{mg} / \mathrm{dL}$ submeteram-se a nova dosagem de glicemia capilar em outra ocasião. Os indivíduos com glicemia de jejum entre $100 \mathrm{mg} / \mathrm{dL}$ e 125 $\mathrm{mg} / \mathrm{dL}$ foram submetidos ao TOTG. Os diagnósticos de DM e tolerância à glicose diminuída foram baseados nos critérios da Associação Americana de Diabetes (18). Desse modo, foram considerados diabéticos os sujeitos que apresentaram glicemia de jejum com nível igual ou acima de $126 \mathrm{mg} / \mathrm{dL}$ em duas ocasiões diferentes, ou glicemia igual ou acima de $200 \mathrm{mg} / \mathrm{dL} 2$ horas após ingestão de $75 \mathrm{~g}$ de glicose anidra no TOTG, além daqueles com diagnóstico prévio de DM. Foram considerados com tolerância à glicose diminuída aqueles que no TOTG apresentaram glicemia de $140 \mathrm{mg} / \mathrm{dL}$ a 199 mg/dL 2 horas após ingestão da glicose anidra.

O peso, em quilogramas, foi aferido com o indivíduo trajando roupa de uso habitual, sem calçados, em balança digital para adultos, com peso limitado em $180 \mathrm{~kg}$, bivolt, com bateria, estrutura tu- 
bular e base de borracha antiderrapante. A altura, em centímetros, foi medida com o indivíduo em pé, sem calçados, em um estadiômetro portátil de estrutura de alumínio, com capacidade de aferição mínima de $0,80 \mathrm{~m}$ e máxima de 2,20 m. O índice de massa corporal (IMC) foi calculado pela fórmula IMC = peso em $\mathrm{kg} /$ quadrado da altura em metros.

A circunferência abdominal foi aferida com fita métrica inelástica, posicionada no ponto médio entre a borda inferior da última costela e a parte superior da crista ilíaca, com o paciente em pé, e registrada em centímetros. Considerou-se normal um perímetro abdominal de até $90 \mathrm{~cm}$ em homens e $80 \mathrm{~cm}$ em mulheres. Esses são os critérios recomendados pela Federação Internacional de Diabetes para as populações sul-americanas, chinesa e do sul da Ásia (19). Tomando como pressuposto que os indígenas das Américas tiveram origem em povos asiáticos que cruzaram o estreito de Behring, reforçase a utilização desses valores para a população da Aldeia Jaguapiru (20).

A pressão arterial foi aferida com esfigmomanômetro aneroide para adultos, com fecho de metal, usado no braço direito do participante sentado, após repouso de 10 minutos. $\mathrm{O}$ valor final foi a média aritmética de duas aferições consecutivas. Quando as pressões arteriais sistólica ou diastólica obtidas apresentaram diferença maior do que $4 \mathrm{mmHg}$, procedeu-se a uma terceira aferição, sendo desconsiderada a mais discrepante. Foram considerados hipertensos os indivíduos que apresentaram pressão sistólica $\geq 140 \mathrm{mmHg}$ e pressão diastólica $\geq 90 \mathrm{mmHg}$ (21).

\section{Análise estatística}

As variáveis qualitativas foram representadas por frequência absoluta (no.) e relativa (\%), e as quantitativas por média e desvio padrão. Utilizaram-se os testes do qui-quadrado $\left(\chi^{2}\right)$ ou exato de Fisher para comparar variáveis qualitativas. A análise de variância de Kruskal-Wallis foi utilizada para comparar o IMC dos grupos com DM e tolerância à glicose diminuída e dos negativos para essa condição.

O projeto foi aprovado pelo Comitê de Ética em Pesquisa do Centro Universitário da Grande Dourados (UNIGRAN) e pela Comissão Nacional de Ética em Pesquisa (CONEP), e obteve parecer de mé- rito técnico-científico do Conselho $\mathrm{Na}$ cional de Desenvolvimento Científico e Tecnológico (CNPq). Os sujeitos que concordaram em participar da pesquisa assinaram um termo de consentimento livre e esclarecido.

\section{RESULTADOS}

Dos 612 participantes previstos, quatro não foram encontrados no domicílio no dia determinado para a dosagem da glicemia de jejum e dois desistiram de participar do estudo. Entre os 606 incluídos, a média de idade foi de $36 \pm 13,5$ anos (extremos de 18 e 69 anos), sendo 338 mulheres $(55,8 \%)$ e 268 homens $(44,2 \%)$. Essa amostra aleatória simples correspondeu a $23,5 \%$ da população de 18 a 69 anos da Aldeia. A distribuição por faixa etária dos participantes do estudo foi semelhante à da população geral da Aldeia Jaguapiru, com amplo predomínio da população jovem.
Observa-se na tabela 1 que a maioria dos participantes apresentava glicemia normal (82\%). A prevalência de DM ajustada para idade foi de $0,81 \%$ na faixa de 18 a 29 anos e de $12,8 \%$ na faixa de 60 a 69 anos. Entre os 12 participantes que receberam o diagnóstico de diabetes durante o estudo, um foi diagnosticado pelo TOTG e 11 apresentaram glicemia de jejum acima de $125 \mathrm{mg} / \mathrm{dL}$ em duas ocasiões. Dos 15 diabéticos com diagnóstico anteriormente estabelecido, somente um apresentava o nível de glicemia controlado. Apenas um diabético fazia uso de insulina. Entre os diabéticos, houve predomínio das mulheres. Quase metade dos participantes diabéticos desconheciam sua condição.

A tabela 2 mostra a distribuição pelo IMC, por sexo e para o grupo como um todo, de acordo com a classificação obtida pela medida da glicemia capilar. Considerando-se o valor de IMC superior a 30, as mulheres com tolerância à

TABELA 1. Distribuição da população com 18 a 69 anos de idade na Aldeia Jaguapiru de acordo com a glicemia capilar em jejum, Dourados (MS), Brasil, 2008

\begin{tabular}{|c|c|c|c|c|c|c|}
\hline \multirow[b]{2}{*}{ Variável } & \multicolumn{2}{|c|}{ Homens } & \multicolumn{2}{|c|}{ Mulheres } & \multicolumn{2}{|c|}{ Total } \\
\hline & No. & $\%$ & No. & $\%$ & No. & $\%$ \\
\hline Glicemia normal & 227 & 84,7 & 270 & 79,9 & 497 & 82 \\
\hline Glicemia de jejum alterada & 34 & 12,7 & 35 & 10,4 & 69 & 11,4 \\
\hline Diabetes melito & 4 & 1,5 & 23 & $6,8^{b}$ & 27 & 4,5 \\
\hline Tolerância à glicose diminuída & 3 & 1,1 & 10 & $2,9^{b}$ & 13 & 2,1 \\
\hline Total & 268 & 44,2 & 338 & 55,8 & 606 & 100 \\
\hline
\end{tabular}

a Testes do qui-quadrado ou exato de Fisher.

b $P=0,005$ em relação ao sexo masculino.

TABELA 2. Distribuição do índice de massa corporal em relação às variações de glicemia dos indígenas em mulheres e homens da Aldeia Jaguapiru, Dourados (MS), Brasil, 2008

\begin{tabular}{|c|c|c|c|c|c|c|c|c|c|c|}
\hline \multirow[b]{2}{*}{$\operatorname{IMC}\left(\mathrm{kg} / \mathrm{m}^{2}\right)^{\mathrm{a}}$} & \multicolumn{2}{|c|}{$\begin{array}{l}\text { Glicemia } \\
\text { de jejum } \\
\text { alterada }\end{array}$} & \multicolumn{2}{|c|}{$\begin{array}{l}\text { Tolerância } \\
\text { à glicose } \\
\text { diminuída }\end{array}$} & \multicolumn{2}{|c|}{$\begin{array}{c}\text { Diabetes } \\
\text { melito }\end{array}$} & \multicolumn{2}{|c|}{$\begin{array}{c}\text { Glicemia } \\
\text { normal }\end{array}$} & \multicolumn{2}{|c|}{ Total } \\
\hline & No. & $\%$ & No. & $\%$ & No. & $\%$ & No. & $\%$ & No. & $\%$ \\
\hline \multicolumn{11}{|l|}{ Homens } \\
\hline$\geq 30$ & 10 & 29,4 & - & - & - & - & 28 & 11,9 & 38 & 14,2 \\
\hline$\geq 25 \mathrm{e}<30$ & 16 & 47 & 3 & $100^{\mathrm{b}}$ & 4 & $100^{c}$ & 95 & 41,8 & 118 & 44 \\
\hline$<25$ & 8 & 23,5 & - & - & - & - & 104 & 45,8 & 112 & 41,8 \\
\hline \multicolumn{11}{|l|}{ Mulheres } \\
\hline$\geq 30$ & 13 & 37,1 & 6 & $60^{d}$ & 12 & 52,2 & 73 & 27,1 & 104 & $30,8^{e}$ \\
\hline$\geq 25$ e $<30$ & 15 & 42,9 & 1 & 10 & 10 & 43,5 & 96 & 35,5 & 122 & 36,1 \\
\hline$<25$ & 7 & 20 & 3 & 30 & 1 & 4,3 & 101 & 37,4 & 112 & 33,1 \\
\hline \multicolumn{11}{|l|}{ Ambos os sexos } \\
\hline$\geq 30$ & 23 & 33,3 & 6 & 46,2 & 12 & 44,4 & 101 & 20,3 & 142 & 23,4 \\
\hline$\geq 25 e<30$ & 31 & 44,9 & 4 & 30,8 & 14 & 51,9 & 191 & 38,4 & 240 & 39,6 \\
\hline$<25$ & 15 & 21,7 & 3 & 23,1 & 1 & 3,7 & 205 & 41,2 & 224 & 36,9 \\
\hline
\end{tabular}

a IMC = índice de massa corporal; testes do qui-quadrado ou exato de Fisher.

b $P=0,02$ em relação a glicose normal.

c $P<0,006$ em relação a glicose normal.

d $P=0,003$ em relação aos demais grupos.

e $P<0,05$ em relação aos homens. 
glicose diminuída tiveram maior percentual de obesidade em relação aos demais grupos $(P<0,05)$. Observou-se, ainda, que um terço das mulheres apresentou IMC menor que 25. Entre os homens, não se verificou obesidade naqueles com tolerância à glicose diminuída nem nos diabéticos. Contudo, esses dois grupos tiveram maiores percentuais de sobrepeso em relação ao grupo com glicemia normal $(P=0,02)$. Observou-se, também, que as mulheres eram mais obesas do que os homens $(P<0,05)$. A obesidade relacionou-se a um aumento de 2,2 vezes para o risco de desenvolver DM.

Nos indivíduos diabéticos, os valores de circunferência abdominal superiores a $80 \mathrm{~cm}$ nas mulheres $(P<0,02)$ e $90 \mathrm{~cm}$ nos homens $(P<0,05)$, além de hipertensão arterial $(P<0,05)$, foram significativamente mais frequentes do que nos demais grupos (tabela 3). Somente uma mulher diabética teve circunferência abdominal com medida inferior a $80 \mathrm{~cm}$.

A história familiar de DM foi referida por 33,3\% dos diabéticos e 30,7\% dos intolerantes à glicose, sendo estatisticamente significativa nesses grupos em relação ao grupo com glicemia normal $(P=0,02)$. Não houve predominância de história materna.

Não se encontrou diferença estatística entre os grupos com e sem diabetes relativamente a tabagismo e história familiar de doença hipertensiva. Declararam-se fumantes 115 indígenas $(18,9 \%)$, porém somente $10 \%$ dos diabéticos e intolerantes à glicose fizeram referência ao tabagismo (tabela 3).

TABELA 3. Fatores de risco cardiometabólicos entre os indígenas da Aldeia Jaguapiru, Dourados (MS), Brasil, 2008

\begin{tabular}{|c|c|c|c|c|c|c|c|c|}
\hline \multirow[b]{3}{*}{ Indicador ${ }^{a}$} & \multicolumn{8}{|c|}{ Glicemia } \\
\hline & \multicolumn{2}{|c|}{ Alterada } & \multicolumn{2}{|c|}{$\begin{array}{l}\text { Tolerância } \\
\text { diminuída }\end{array}$} & \multicolumn{2}{|c|}{$\begin{array}{c}\text { Diabetes } \\
\text { melito }\end{array}$} & \multicolumn{2}{|c|}{ Normal } \\
\hline & $n=69$ & $\%$ & $n=13$ & $\%$ & $n=27$ & $\%$ & $n=497$ & $\%$ \\
\hline \multicolumn{9}{|l|}{ Circunferência abdominal } \\
\hline Homens $^{b}$ & 23 & 33,3 & 3 & 23 & 4 & $100^{c}$ & 80 & 16 \\
\hline Mulheres $^{d}$ & 30 & 43,5 & 8 & 61,5 & 22 & $81,5^{c}$ & 199 & 40 \\
\hline $\begin{array}{l}\text { Hipertensão arterial } \\
\text { História familiar de }\end{array}$ & 24 & 34,8 & 5 & 38,5 & 22 & $81,5^{\mathrm{e}}$ & 129 & 26 \\
\hline $\begin{array}{l}\text { doença hipertensiva } \\
\text { História familiar de }\end{array}$ & 34 & 49,3 & 8 & 61,5 & 19 & 70,3 & 260 & 52,3 \\
\hline diabetes melito & 26 & 37,7 & 4 & 30,7 & 9 & 33,3 & 110 & $22,1^{f}$ \\
\hline Tabagismo & 14 & 20,3 & 1 & 7,7 & 3 & 11,1 & 97 & 19,5 \\
\hline \multicolumn{9}{|c|}{$\begin{array}{l}\text { a Testes do qui-quadrado ou exato de Fisher. } \\
\text { b }>90 \mathrm{~cm} \text {. } \\
\text { c } P<0,007 \text { em relação aos outros grupos. } \\
\text { d }>80 \mathrm{~cm} \text {. } \\
\text { e } P<0,05 \text { em relação aos outros grupos. } \\
\text { f } P=0,02 \text { em relação aos outros grupos. }\end{array}$} \\
\hline
\end{tabular}

Observamos ainda que $12,5 \%$ dos participantes não eram alfabetizados, $66,3 \%$ não haviam cursado o ensino médio e $15 \%$ haviam completado o ensino médio ou o curso superior. O percentual de mulheres diabéticas e intolerantes à glicose não alfabetizadas foi significativamente maior em comparação às não diabéticas $(P<0,05)$.

$\mathrm{O}$ rendimento mensal familiar declarado foi menor do que 1 salário mínimo ( $\mathrm{R} \$ 415,00$ na época do estudo) em 64,6\% das famílias pesquisadas; $24,5 \%$ das famílias recebiam entre 1 e 2 salários. Apenas 9,9\% recebiam acima de 2 salários mínimos. A prevalência de DM e tolerância à glicose diminuída não se associou à renda familiar.

O emprego no comércio ou nas lavouras de cana-de-açúcar da região foi apontado como a principal atividade econômica dos indígenas do sexo masculino; outra atividade referida, embora não principal, foi o trabalho em lavouras próprias, que visam à autossubsistência. Entre as mulheres, uma parcela estava empregada nos centros de saúde ou nas escolas da reserva indígena ou, ainda, como domésticas na Cidade de Dourados. Os afazeres domésticos foram a atividade predominante entre as mulheres.

\section{DISCUSSÃO}

No presente estudo, verificou-se que o DM e a tolerância à glicose diminuída estiveram presentes, respectivamente, em 4,5 e 2,1\% dos indivíduos de 18 a 69 anos de idade na população indígena da Al-

deia Jaguapiru. Essas prevalências são inferiores às encontradas no estudo multicêntrico brasileiro, realizado no período de 1986 a 1988, que registrou taxas de 7,6 e $8,7 \%$, respectivamente (5). Devese, porém, levar em consideração que o estudo multicêntrico brasileiro foi realizado em grandes cidades, nas quais a prevalência de DM e de tolerância à glicose diminuída tem se mostrado superior à da zona rural.

Outra possível explicação para essa diferença é a abrangência da população em relação à faixa etária: enquanto no estudo multicêntrico a população partia dos 30 anos, neste estudo a delimitação de idade partiu dos 18 anos. Isso é sugerido pelo fato de que a prevalência de DM foi baixa $(0,81 \%)$ nos jovens com menos de 29 anos, mas aumentou substancialmente $(12,8 \%)$ nos indivíduos de 60 a 69 anos, fato semelhante ao descrito na cidade de Campos dos Goytacazes, Rio de Janeiro (22). Essa observação está de acordo com as constatações de que o envelhecimento da população se associa ao aumento da frequência de DM (4), também entre os indígenas. Entretanto, a prevalência observada por nós se aproximou da taxa de $3,4 \%$ encontrada em uma comunidade rural brasileira (23), enquanto foi superior aos $2,2 \%$ relatados na comunidade terena da Aldeia de Buriti, em Mato Grosso do Sul (17). Também foi superior à taxa descrita na $\mathrm{Al}$ deia de Sangradouro-Volta Grande (Estado de Mato Grosso), onde somente cinco casos de DM do tipo 2 foram observados entre 590 indígenas xavante (13), e, ainda, na comunidade ianomâmi, com apenas um caso da doença em 72 adultos (11). Em comunidades indígenas do Alto Xingu, entre 201 indígenas de ambos os sexos e com idade acima de 20 anos, nenhum caso de DM foi observado, mas foram relatados oito $(4,6 \%)$ casos de glicemia de jejum alterada (24).

Comparando-se os dados do presente estudo com os de populações indígenas de outros países, verifica-se que os mesmos se aproximam dos 4,4\% de DM tipo 2 entre os índios otomi, no México (8), e da prevalência de 6,7\% encontrada em comunidades aborígenes australianas (9); no entanto, estão muito abaixo da prevalência de quase $50 \%$ observada em indígenas com idade acima de 35 anos em comunidades norte-americanas (25).

A ampla variação de prevalência de DM e de tolerância à glicose diminuída nas diversas populações estudadas pode 
ser decorrente de fatores genéticos e ambientais característicos de cada comunidade. Por exemplo, em comunidades aborígenes do Havaí (26), foram descritas diferentes taxas de prevalência de DM de acordo com o grau de integração à cultura ocidental: $10 \%$ para os nativos isolados e $27,9 \%$ para os aculturados, índices muito superiores aos verificados neste trabalho.

No presente estudo, muitos dos diabéticos $(44,4 \%)$ desconheciam sua condição de portadores da doença. Fato semelhante foi relatado no estudo multicêntrico brasileiro (46\%) (5) e entre norteamericanos $(39,3 \%)$ nos anos de 2003 a 2004 (27). Todos os indivíduos diagnosticados em nosso estudo receberam orientação sobre alimentação e atividade física e foram encaminhados para atendimento no serviço especializado, sob os cuidados do coordenador da pesquisa.

$\mathrm{O}$ risco de desenvolvimento de $\mathrm{DM}$ tipo 2 está estreitamente ligado à presença e à duração de sobrepeso e obesidade. A literatura mostra que $90 \%$ dos diabéticos do tipo 2 têm sobrepeso ou obesidade (28). No presente estudo, o IMC esteve acima de 25 em 96,3\% dos diabéticos e em 76,9\% dos indígenas com tolerância à glicose diminuída, como já foi descrito para uma população de país desenvolvido (3). Além disso, a obesidade relacionou-se a um aumento de 2,2 vezes no risco de desenvolver $\mathrm{DM}$, à semelhança do que foi relatado em uma população de mulheres brancas (29) e na população de Ribeirão Preto (30).

Entre as mulheres da Aldeia Jaguapiru, 30,8\% apresentaram IMC acima de 30 , uma porcentagem inferior $(41,3 \%)$ àquela observada em mulheres da comunidade xavante de São José, Estado de Mato Grosso (31). Porém, esse valor superou os 10,9\% registrados em uma comunidade rural em Minas Gerais (23), bem como o percentual de $12,5 \%$ encontrado entre os indígenas do Alto Xingu (24), os 18,7\% descritos na Aldeia Buriti (17) e os $12,5 \%$ verificados na comunidade indígena Parkatêjê, Estado do Pará (32). Nessa última comunidade indígena, diferentemente do encontrado na Aldeia Jaguapiru, os níveis de IMC mais elevados no sexo feminino concentraram-se na faixa etária de 50 a 59 anos. É possível que o aumento do IMC nas mulheres jovens possa refletir maior assimilação dos hábitos ocidentais e a perda da cultura tradicional indígena nessa faixa etária.
$\mathrm{O}$ registro de obesidade entre os homens da Aldeia foi menor do que nas mulheres, já que somente $14,2 \%$ tiveram $I M C \geq 30$. Esse percentual é um pouco superior aos $11,6 \%$ da comunidade terena de Buriti (MS) (17), pouco menor do que os $18,2 \%$ registrados na comunidade do Alto Xingu (24) e menor do que os $24,6 \%$ verificados entre os homens da comunidade xavante de São José (31). Entretanto, é muito superior à cifra de 1,4\% encontrada em uma comunidade rural mineira (23), bem como ao percentual de $1,7 \%$ na comunidade indígena Parkatêjê (PA) (32). Os valores de IMC nos homens da Aldeia Jaguapiru devem ser avaliados com reserva, já que, na sua grande maioria, esses homens são trabalhadores braçais, com massa muscular bem desenvolvida. Portanto, valores mais elevados de IMC podem não representar aumento de tecido gorduroso, mas, em vez disso, ganho de massa magra.

A adiposidade intra-abdominal é a que tem maior impacto sobre a resistência à insulina (33). Na população diabética, ou naquela com tolerância à glicose diminuída, observou-se que 90,9\% das mulheres tinham perímetro abdominal maior que $80 \mathrm{~cm}$. Todos os homens encontravam-se acima da medida de 90 $\mathrm{cm}$. Além disso, 70,4\% das mulheres e $41 \%$ dos homens da Aldeia tiveram circunferência abdominal maior do que o preconizado para cada sexo, o que os classifica como portadores de fatores de risco cardiometabólicos. Para o grupo de mulheres, o percentual obtido para essa variável foi semelhante aos $86,9 \%$ descritos no estudo dos guaranis mbyás do Estado do Rio de Janeiro (34). Nos homens, porém, foi significativamente superior aos 6,5\% encontrados no mesmo estudo (34). Pode-se associar esse achado à prática do trabalho braçal em lavouras do entorno da Aldeia entre os homens quando comparados às mulheres; porém, o presente estudo não permite confirmar esse dado.

A hipertensão arterial manifestou-se em 29,7\% dos participantes, sem diferença estatística entre os sexos. Esse percentual é superior aos 4,8\% descritos em índios guarani mbyá no litoral do Rio de Janeiro (34), bem como aos $11,1 \%$ dos terena da Aldeia Buriti (MS) (23), mas é inferior aos $37,7 \%$ relatados em indígenas do Alto Xingu (24).

O DM é considerado fator de risco independente para doença cardiovascular. Porém, frequentemente, está associado à obesidade e à hipertensão arterial (35). Na Aldeia Jaguapiru, 67,5\% dos diabéti$\cos$ e dos portadores de tolerância à glicose diminuída tinham hipertensão arterial. Em estudo multicêntrico, em pacientes diabéticos no Rio Grande do Sul, a hipertensão arterial foi relatada em $77,6 \%$ dos homens e em 83,3\% das mulheres (36). Tal achado foi semelhante aos $81,5 \%$ encontrados entre os diabéti$\cos$ da Aldeia Jaguapiru que participaram da pesquisa e aos $77 \%$ de hipertensos entre os adultos diabéticos da tribo pima (25). Possivelmente, esses dois grupos indígenas, com fatores genéticos diversos, estejam experimentando mudanças similares no que se refere ao hábito alimentar e ao modo de vida.

A história familiar de DM é descrita como importante evento preditor de risco para o desenvolvimento de DM tipo 2 (37), sendo que, no presente trabalho, essa foi referida em $33,3 \%$ dos diabéticos e em 30,7\% dos intolerantes à glicose. Crispin et al. (38), no Sul do Brasil, reportaram ao menos um membro de primeiro grau da família afetado pela doença em 76,6\% de 644 diabéticos do tipo 2, havendo predominância de história materna. Entretanto, no presente estudo, não se verificou diferença entre a história familiar materna e paterna de DM entre os indígenas acometidos pela doença.

O estresse psicológico pode desempenhar um papel importante no desenvolvimento do DM (39). Pode-se supor que os indígenas da Aldeia Jaguapiru, confinados em uma pequena extensão de terras degradadas e sem a tecnologia necessária para retirar da terra o seu sustento, estejam expostos a estresse, fator que se associou à ocorrência de 34 suicídios de índios da etnia guarani e kaiowá no Mato Grosso do Sul, em 2008 (40). Embora o presente estudo não tenha tido o objetivo de avaliar a presença de estresse nessa população indígena, esse fator não parece ter influenciado o desenvolvimento de DM, visto que a prevalência de DM não foi maior do que a verificada na população em geral.

O tabagismo foi declarado por 115 indígenas (19\%) dos 606 avaliados, o que é inferior aos $51 \%$ referidos no estudo realizado na Aldeia Buriti (17) e um pouco superior $(14,9 \%)$ ao encontrado na população brasileira (41). Porém, somente $10 \%$ dos indígenas diabéticos ou com tolerância à glicose diminuída referiram tabagismo, o que não está de acordo com 
a proposição de aumento da prevalência de DM entre homens fumantes (42). Entretanto, não se pode ignorar que a falta de associação pode refletir mudanças de hábitos decorrentes do diagnóstico prévio do DM.

Não foi evidenciada associação entre renda familiar e DM e tolerância à glicose diminuída neste estudo. Provavelmente, isso ocorreu porque as condições de vida na Aldeia e a renda familiar dos indígenas não tinham a discrepância observada nas sociedades modernas em outras comunidades. Neste estudo, registraram-se apenas 9,9\% de famílias que recebiam acima de 2 salários mínimos. Saad (17) também relatou renda per capita abaixo de meio salário mínimo em $70 \%$ dos indígenas da Aldeia Buriti, $26,1 \%$ com meio a 1 salário e $3,75 \%$ acima de 1 salário mínimo.

O percentual de não alfabetizados foi significativamente maior entre os diabéticos e os indivíduos com tolerância à glicose diminuída em relação aos não diabéticos, dados semelhantes aos da população brasileira (5). O nível de escolaridade registrado foi baixo, pois $78,8 \%$ dos sujeitos da amostra não tinham ingressado no ensino médio. Saad (17) também observou baixa escolaridade da comunidade terena de Buriti, na qual 69,6\% tinham até no máximo 4 anos de estudo.
Entre as limitações deste estudo está a inclusão de indígenas de três etnias, guarani, kaiowá e terena, em diferentes fases de assimilação da cultura ocidental. Como visto anteriormente, podem ocorrer prevalências diferentes entre etnias. Outra limitação deve-se ao caráter transversal do estudo, em que o diagnóstico da situação epidemiológica é um retrato estático do momento, fornecendo evidências fracas de causa e efeito, não permitindo a extrapolação dos resultados com segurança para outras situações. A distribuição dos participantes por sexo também não corresponde à da população da Aldeia na faixa etária do estudo. Isso pode ser decorrência do trabalho temporário desenvolvido pelos homens em usinas de açúcar no entorno da Aldeia, que determina a sua ausência por períodos às vezes maiores do que 1 mês. Outra limitação foi a inclusão de mais de um participante da mesma família, permitindo que fatores genéticos pudessem interferir na prevalência do diabetes na comunidade avaliada. Finalmente, é importante notar que, por tratar-se de pesquisa em campo, utilizamos a dosagem da glicemia capilar e não a glicemia plasmática, padrão ouro no diagnóstico de DM.

Em conclusão, a prevalência de DM e de tolerância à glicose diminuída na população de 18 a 69 anos de idade da Al- deia Jaguapiru é inferior à da população brasileira; a prevalência de obesidade foi elevada, sendo que as mulheres apresentaram maior risco para obesidade e DM do que os homens. No entanto, a prevalência da hipertensão arterial foi semelhante à da população brasileira, não havendo diferença entre os sexos. O controle glicêmico e pressórico dos indígenas com diagnóstico estabelecido de DM e hipertensão arterial foi inadequado.

A prevalência de DM tipo 2 tem aumentado mundialmente como resultado do envelhecimento da população, da urbanização, da inatividade física e do aumento da obesidade. Os dados aqui descritos sugerem que esses fatores também contribuem para o aumento da prevalência de DM tipo 2 na Aldeia Jaguapiru. Os resultados deste estudo sugerem a necessidade de orientações nutricionais que visem ao estímulo do consumo de cereais integrais, frutas e verduras, e do incentivo à prática de atividades físicas entre os indígenas da Aldeia Jaguapiru como forma de prevenção do DM.

Agradecimentos. Agradecemos aos alunos da Faculdade de Ciências da Saúde da Universidade Federal da Grande Dourados, componentes da Liga de Diabetes Mellitus, pela colaboração na coleta de dados.

\section{REFERÊNCIAS}

1. Forti A, Loureiro R, Gusmão A. Diabetes mellitus: classificação e diagnóstico. Em: Vilar L. Endocrinologia clínica. $3^{\mathrm{a}}$ ed. Rio de Janeiro: Guanabara Koogan; 2006. Pp. 539-48.

2. Winer N, Sowers J. Epidemiology of diabetes. J Clin Pharmacol. 2004;44(4):397-405.

3. Hossain P, Katar B, El Nahas M. Obesity and diabetes in the developing world-a growing challenge. N Engl J Med. 2007;356(63):213-5.

4. Wild S, Roglic G, Green A, Sicree R, King H. Global prevalence of diabetes: estimates for the year 2000 and projections for 2030. Diabetes Care. 2004;27(5):1047-53.

5. Malerbi DA, Franco LJ. Multicenter study of the prevalence of diabetes mellitus and impaired glucose tolerance in the urban Brazilian population aged 30-69 yr. Diabetes Care. 1992;15(11):1509-16.

6. Yeates K, Tonelli M. Indigenous health: update on the impact of diabetes and chronic kidney disease. Curr Opin Nephrol Hypertens. 2006;15(6):588-92.

7. Gohdes D, Kaufman S, Valway S. Diabetes in American Indians. An overview. Diabetes Care. 1993;16(1):239-43.
8. Alvarado-Osuna C, Milian-Suazo F, VallesSanchez V. Prevalencia de diabetes mellitus e hiperlipidemias en indígenas otomíes. Salud Publica Mex. 2001;43(5):459-63.

9. Australian Institute of Health and Welfare. The health and welfare of Australia's aboriginal and Torres Strait Islander peoples 2005. Canberra: Australian Bureau of Statistics and AIHW; 2005. Disponível em: www.aihw.gov. au/WorkArea/DownloadAsset.aspx?id=644 2458575. Acessado em 5 de abril de 2008.

10. Vieira Filho JPB. O diabetes mellitus e as glicemias de jejum dos índios Caripuna e Palikur. Rev Assoc Med Bras. 1977;23(5): 175-8.

11. Bloch KV, Coutinho ESF, Lobo MSC. Pressão arterial, glicemia capilar e medidas antropométricas em uma população Yanomámi. Cad Saude Publica. 1993;9(4):428-38.

12. Tavares EF, Vieira Filho JPB, Andriolo A, Franco LJ. Anormalidades de tolerância à glicose e fatores de risco cardiovascular em uma tribo indígena aculturada da região amazônica brasileira. Arq Bras Endocrinol Metabol. 1999;43(Supl 1):235-9.
13. Vieira Filho JPB. Emergência do diabetes melito tipo 2 entre os Xavántes. Rev Assoc Med Bras. 1996;42(1):61-5.

14. Rocha AKS, Bós AJG, Huttner E, Machado DC. Prevalência da síndrome metabólica em indígenas com mais de 40 anos no Rio Grande do Sul, Brasil. Rev Panam Salud Publica. 2011;29(1):41-5.

15. Coimbra Junior CEA, Santos RV, Escobar AL. Epidemiologia e saúde dos povos indígenas no Brasil. Rio de Janeiro: Fiocruz/ABRASCO; 2003.

16. Brasil, Fundação Nacional de Saúde. Distrito sanitário de Mato Grosso do Sul. Disponível em: http://sis.funasa.gov.br/transparencia publica/siasiweb/Layout/quantitativo de_pessoas_2008.asp. Acessado em 18 de fevereiro de 2008.

17. Saad MBNL. Saúde e nutrição terena: sobrepeso e obesidade [dissertação]. Campo Grande: Universidade Federal de Mato Grosso do Sul; 2005

18. Genuth S, Alberti KG, Bennett P. Follow-up report on the diagnosis of diabetes mellitus. Diabetes Care. 2003;26(3):160-7. 
19. International Diabetes Federation. The IDF consensus worldwide definition of the metabolic syndrome. Disponível em: www.idf. org/webdata/docs/Metac_syndrome_def.p df. Acessado em 20 de janeiro de 2009.

20. Brasil, Fundação Nacional do Índio (FUNAI). A origem dos povos americanos. Disponível em: http://www.funai.gov.br/home/menu2. php. Acessado em 25 de agosto de 2008.

21. Sociedade Brasileira de Cardiologia. V Diretrizes Brasileiras de Hipertensão Arterial. Arq Bras Cardiol. 2007;89(3):24-79.

22. Souza JL, Chalita FEB, Reis AFF, Teixeira CL, Gicovate CN, Bastos DA, et al. Prevalência de diabetes mellitus e fatores de risco em Campos dos Goytacazes, RJ. Arq Bras Endocrinol Metab. 2003;47(1):69-74.

23. Silva DA, Felisbino-Mendes MS, Pimenta AM, Gazzinelli A, Hac G, Velasquez-Melendez G. Distúrbios metabólicos e adiposidade em uma população rural. Arq Bras Endocrinol Metab. 2008;53(3):489-98.

24. Gimeno SGA, Rodrigues D, Pagliaro H, Cano EM, Lima EES, Baruzzi RG. Perfil metabólico e antropométrico de índios Aruák: Mehináku, Waurá e Yawalapití, Alto Xingu, Brasil Central, 2000/2002. Cad Saude Publica. 2007; 23(8):1946-54.

25. Pavkov ME, Knowler WC, Bennett PH, Looker HC, Kradoff J, Nelson RG. Increasing incidence of proteinuria and declining incidence of end-stage renal disease in diabetic Pima Indians. Kidney Int. 2006;70(10):1840-6.

26. Kaholokula JK, Nacapoy AH, Grandinetti A, Chang HK. Association between acculturation modes and type 2 diabetes among Native Hawaiians. Diabetes Care. 2008;31(4): 698-700.

27. American Diabetes Association. Economic cost of diabetes in the US in 2007. Diabetes Care. 2008;31(3):596-615.
28. Must A, Spadano J, Coakley EH, Field AE, Colditz G, Dietz WH. The disease burden associated with overweight and obesity. JAMA. 1999;282(16):1523-9.

29. Colditz GA, Willett WC, Rotnitzky A, Manson JE. Weight gain as a risk factor for clinical diabetes mellitus in women. Ann Intern Med. 1995;122(7):481-6.

30. Torquato MT, Montenegro Junior RM, Viana LA, de Souza RA, Lanna CM, Lucas JC, et al. Prevalence of diabetes mellitus and impaired glucose tolerance in the urban population aged 30-69 years in Ribeirão Preto (São Paulo), Brazil. Sao Paulo Med J. 2003;12(6): 224-30.

31. Gugelmin SA, Santos RV. Ecologia humana e antropometria nutricional de adultos Xavántes, Mato Grosso, Brasil. Cad Saude Publica. 2001;17(2):313-22.

32. Capelli JCS, Koifman S. Avaliação do estado nutricional da comunidade indígena Parkatêjê, Bom Jesus do Tocantins, Pará, Brasil. Cad Saude Publica. 2001;17(2):433-7.

33. Wajchenberg BL. Subcutaneous and visceral adipose tissue: their relation to the metabolic syndrome. Endocr Rev. 2000;21(6):697-738.

34. Cardoso AM, Mattos IE, Koifman RJ. Prevalência de fatores de risco para doenças cardiovasculares na população GuaraniMbyá do Estado do Rio de Janeiro. Cad Saude Publica. 2001;17(2):345-54.

35. Gerstein HC, Pogue J, Mann JFE, Lonn E, Dagenais GR, McQueen M, et al. The relationship between dysglycemia and cardiovascular and renal risk in diabetic and nondiabetic participants in the HOPE study: a prospective epidemiological analysis. Diabetologia. 2005; 48(9):1749-55.

36. Picon PX, Zanatta CM, Gerchman F, Zelmanovitz T, Gross JL, Canani LH. Análise dos critérios de definição da síndrome metabólica em pacientes com diabetes melito tipo 2. Arq Bras Endocrinol Metab. 2006; 550(2):250-63.

37. Meigs JB, Cupples A, Wilson PWF. Parental transmission of type 2 diabetes: the Framingham Offspring Study. Diabetes. 2000;49(12): 2201-7.

38. Crispin D, Canani LH, Gross JL, Tschiedel B, Souto KEP, Roisenberg I. História familiar de diabetes tipo $2 \mathrm{em}$ pacientes do sul de Brasil e sua influência nas características dessa doença. Arq Bras Endocrinol Metab. 2006; 50(5):862-8.

39. Surwit RS, Schneider MS, Feinglos MN. Stress and diabetes mellitus. Diabetes Care. 1992;15(10):1413-22.

40. Conselho Indigenista Missionário. Relatório de violência/2008. Disponível em: www. cimi.org.br/?system $=$ news\&action $=$ read\&id $=3808 \&$ eid $=397$. Acessado em 10 de fevereiro de 2009.

41. Brasil, Ministério da Saúde, Instituto Nacional de Câncer. Inquérito nacional sobre tabagismo. Disponível em: http://bvsms. saude.gov.br/bvs/publicacoes/tabaco inquerito_nacional_070504.pdf. Acessado em 18 de agosto de 2008.

42. Uchimoto S, Tsumura K, Hayashi T, Suematsu C, Endo G, Fujii S, et al. Impact of cigarette smoking on the incidence of type 2 diabetes mellitus in middle-aged Japanese men: the Osaka Health Survey. Diabet Med. 1999;16(11):951-5.

Manuscrito recebido em 24 de junho de 2010. Aceito em versão revisada em 23 de novembro de 2010.
ABSTRACT

Prevalence of diabetes
mellitus and impaired
glucose tolerance in
indigenous people from
Aldeia Jaguapiru, Brazil

Key words
Objective. To determine the prevalence of diabetes mellitus (DM) and impaired glucose tolerance in indigenous people from Aldeia Jaguapiru, in Dourados, state of Mato Grosso do Sul.

Methods. Between August 2007 and July 2008, individuals aged 18-69 years were evaluated. To obtain the simple random sample for the study, 349 houses were picked from among the total 1255 houses in the village. Pregnant women, nonindigenous individuals, and their descendents, and those using glucocorticoids were excluded from the sample. Six hundred and six people were studied (268 men and 338 women). Capillary glucose was measured with a glucose meter, and the oral glucose tolerance test was performed as necessary.

Results. A 4.5\% prevalence was observed for DM and 2.2\% for impaired glucose tolerance, with higher frequency among women. Among diabetics, $44.4 \%$ had not been previously diagnosed. Obesity was present in $14.2 \%$ of men and $30.8 \%$ of women. The prevalence of hypertension was $29.7 \%$ for the overall group and $67.5 \%$ in diabetics and individuals with impaired glucose tolerance. There was no statistical relationship between smoking and the presence of DM and impaired glucose tolerance.

Conclusions. The prevalence of DM and impaired glucose tolerance was lower in this sample compared to the Brazilian population. However, the prevalence of obesity was higher, and that of hypertension was similar. Nutritional guidance and encouragement of physical activity are recommended in Jaguapiru as preventive measures for DM.

Diabetes mellitus; indigenous population; hypertension; obesity; Indians, South American; Brazil. 